ISSN: $1560-9111$

\section{El mapa conceptual en la comprensión lectora de estudiantes de odontología según estilos de aprendizaje}

The use of conceptual map in reading comprehension for dental students according to learning styles

\begin{abstract}
Resumen:
Objetivo: determinar si el uso del mapa conceptual utilizando Cmap Tools favorece la comprensión lectora de un texto expositivo en estudiantes de Odontología, según estilos de aprendizaje. Método: se realizó un estudio cuasiexperimental con pretest y postest en 50 estudiantes de Odontología de la Universidad Nacional Mayor de San Marcos. Los estilos de aprendizaje fueron clasificados con el cuestionario de Honey-Alonso de Estilos de Aprendizaje (CHAEA), el nivel de comprensión de lectura se determinó con el test de comprensión lectora prediseñado y la rúbrica semántica de Miller-Cańas 2008 se utilizó para la evaluación del contenido del mapa conceptual. Resultados: las comparaciones del pre test y postest de comprensión lectora alcanzaron una puntuación promedio para el total de la muestra de 7,5 incrementándose a 9,3 siendo favorecidos los estilos de aprendizaje teórico y reflexivo. La evaluación semántica del mapa conceptual pasó de 4,5 a 9,1 puntos, aquí todos los estilos fueron favorecidos. Conclusión: se reconoce la importancia de utilizar los mapas conceptuales en la educación superior como parte de la mejora de la comprensión lectora del estudiante.
\end{abstract}

Palabras clave: Comprensión de lectura, tecnología educacional, aprendizaje.

\section{Artí́culo Original}

\section{C.D. Zeira Quinto Márquez'1, Mg. Carmen Quintana del Solar ${ }^{2}$}

\begin{abstract}
Objective: the aim of the study was to determine whether the use of conceptual map CmapTools encourages reading comprehension of expository text in dental students according to learning styles. Methodology: A quasi-experimental study with pretest and posttest in 50 students from Universidad Nacional Mayor de San Marcos was performed. Learning styles were classified with the questionnaire Honey-Alonso Learning Styles (CHAEA), the level of reading comprehension was determined using the reading comprehension predesigned test and the evaluation of the conceptual map content using MillerCańas semantic rubric 2008. Results: The comparisons of pretest and posttest of reading comprehension achieved an average score for the total sample of 7.5 increasing to 9.3 being favored the reflective and theoretical learning styles. The evaluation semantics of the conceptual map went from 4,5 to 9,1 points, here all the styles were favored. Conclusion: it is recognized the importance of using the conceptual map in superior education as part of the improvement of the students' reading comprehension.
\end{abstract} Keywords: Reading comprehension; educational technology; learning
1. Unidad de Posgrado de la Facultad de Odontología de la Universidad Nacional Mayor de San Marcos, Perú

2. Departamento Académico de Estomatología Pediátrica de la Facultad de Odontología de la Universidad Nacional Mayor de San Marcos, Perú.

\section{Correspondencia}

C.D. Zeira Quinto Márquez

Calle 31 No 124 Payet, Lima 28, Perú.

Correo electrónico: magalyquinto_36@yahoo.es

Coautor:

Quintana del Solar: cquintanad@unmsm.edu.pe

\section{Introducción}

La comprensión de un texto expositivo es importante en el alumno a nivel universitario y puede ser favorecida por el uso de los mapas conceptuales como herramientas de organización del conocimiento después de una lectura. El mapa puede ser elaborado con un software gratuito como es el Cmap Tools. Destacando la creatividad, la reflexión el análisis y el aprendizaje significativo que el estudiante desea alcanzar. El mapa conceptual incrementa el nivel de comprensión lectora, independientemente del sentido de representación vertical y horizontal ${ }^{1}$, siendo mucho mejor cuando se utilizan estrategias de lectura y cuando se activan y utilizan los conocimientos previos en la elaboración del mapa conceptual. La reelaboración mejora la estructura y la semántica del mapa conceptual ${ }^{2}$ profundizando en el proceso de comprensión de lectura. Esto va de la mano con la motivación, objetivos y preguntas que se planteen para que se realice la comprensión.

La identificación de la idea principal del texto expositivo permite obtener de la lectura conceptos principales y conceptos secundarios. La apropiación de conceptos principales permite una mejor estructuración de los mapas conceptuales $^{3}$. Las instrucciones precisas y claras hacia los alumnos al momento de elaborar el mapa conceptual con el software Cmap Tools permite menos errores en su proceso de elaboración. Es necesaria mayor práctica de la técnica del Cmap Tools para su entendimiento y favorecimiento de la comprensión lectora del texto. Se considera que a mayor práctica mayor entendimiento de la técnica, así que para comprender un texto y demostrar ese entendimiento en el mapa conceptual es importante entender la técnica del Cmap Tools ${ }^{4}$. 
Este trabajo de investigación tiene como objetivo determinar si el uso del mapa conceptual utilizando el Cmap Tools favorece la comprensión lectora de un texto expositivo en estudiantes de Odontología, según estilos de aprendizaje.

\section{Materiales y método}

Esta investigación cuasiexperimental con pretest y postest se realizó en una muestra de 50 estudiantes ingresantes a la facultad de Odontología 2014 en el curso de Estomatología Integrada I de la UNMSM. Se tomaron en cuenta los siguientes criterios de inclusión: 1) Estudiantes matriculados en el curso de pregrado. 2) Estudiantes que utilicen laptop o computadora para la segunda evaluación de la comprensión lectora con mapa conceptual con Cmap Tools. 3) Estudiantes que hayan participado en el taller de mapas conceptuales. 4) Estudiantes que hayan cumplido todo el proceso de investigación.

Procedimientos: 1) Identificación de los estilos de aprendizaje de los estudiantes del curso de Estomatología Integrada. 2) Se realizó la primera evaluación de la comprensión lectora con un mapa conceptual sin Cmap Tools, que consistió en realizar un resumen, identificar la idea principal, colocar un título, responder tres preguntas acerca del texto leído y realizar un mapa conceptual con conocimientos obtenidos en el colegio. 3) Se realizó un taller acerca de qué son y cómo se elaboran los mapas conceptuales, identificación de la idea principal y errores en las proposiciones encontradas en la primera evaluación. 4) Se efectuó la segunda evaluación de la comprensión lectora realizando un mapa conceptual con Cmap Tools, que consistió en realizar un resumen, identificar la idea principal, colocar un título. responder tres preguntas acerca del texto leído y realizar un mapa conceptual con Cmap Tools representando los conceptos previos, del texto, conceptos metas y conceptos agregados. 5) Comparación de ambas evaluaciones del nivel de comprensión lectora y relacionarla con el nivel de comprensión lectora. Para la medición de las variables se utilizaron los siguientes instrumentos: 1) El cuestionario de estilos de Aprendizaje CHAEA para determinar el estilo de aprendizaje. 2) La rúbrica semántica Miller Cańas 2008 para evaluar el contenido del mapa conceptual. 3) El criterio de calificación del test de comprensión lectora (autoelaborado).

Para el pretest y el postest se consideró la lectura de un artículo de 6 hojas de Accidentes con material biológico (Rev. Clin Med FAM 2011; 4:19-24) durante 20 minutos, para luego ser retirado y proceder con el desarrollo del test de comprensión lectora y elaborar el mapa conceptual con y sin Cmap
Tools. Los resultados fueron tabulados en Excel 2010, luego transferidos al programa SPSS 20 y analizados con la prueba no paramétrica de Wilcoxon con un nivel de significancia $\mathrm{p}<0,05$.

\section{Resultados}

\section{Estilos de aprendizaje}

Los estilos de aprendizaje de los estudiantes de Odontología se clasificaron en: estilo teórico (40\%), en segundo lugar los activos (32\%) y, en menor proporción, el estilo reflexivo (18\%) y pragmático (10\%).

\section{Comprensión lectora con y sin Cmap Tools utilizando el criterio de calificación del test de comprensión lectora}

Sin Cmap Tools, la mayoría alcanzó un nivel regular y la minoría alcanzó un nivel bueno de comprensión lectora. Con el Cmap Tools, la mayoría alcanzó un nivel bueno y la minoría alcanzó un nivel regular, al comparar la comprensión lectora sin y con Cmap Tools.

Se utilizó la prueba no paramétrica de Wilcoxon. Se comparó el pre y el pos test del nivel de comprensión lectora de los cuatro grupos de estudiantes según su estilo. Los resultados mostraron que todos los estilos, a excepción del estilo pragmático, tuvieron un cambio significativo $\mathrm{p}<0,05$ (Tabla 1 ).

Tabla 01. Comparaciones del nivel de comprensión lectora pre y postest de los estudiantes de diferentes estilos de aprendizajes usando el criterio de calificación del test de comprensión lectora

\begin{tabular}{|c|c|c|c|c|}
\hline \multirow{3}{*}{$\begin{array}{l}\text { ESTILOS DE } \\
\text { APRENDIZAJE }\end{array}$} & \multirow{3}{*}{$\begin{array}{l}\text { ESTADÍSTICAS } \\
\text { DESCRIPTIVAS }\end{array}$} & \multicolumn{2}{|c|}{ Nivel de comprensión lectora } & \multirow{3}{*}{$\mathrm{p}\left(^{*}\right)$} \\
\hline & & \multirow{2}{*}{$\begin{array}{l}\text { antes } \\
\text { CmapTools }\end{array}$} & \multirow{2}{*}{$\begin{array}{l}\text { después } \\
\text { CmapTools }\end{array}$} & \\
\hline & & & & \\
\hline \multirow[t]{2}{*}{ ACTIVO } & Mínimo - Máximo & $6-9$ & $7-12$ & 0,002 \\
\hline & Media $\pm D e s v$. Típica & $7,8 \pm 0,98$ & $9,2 \pm 1,2$ & \\
\hline \multirow[t]{2}{*}{ REFLEXIVO } & Mínimo - Máximo & $5-9$ & $7-12$ & 0,007 \\
\hline & Media $\pm D e s v$. Típica & $7,2 \pm 1,3$ & $9,8 \pm 1,6$ & \\
\hline \multirow[t]{2}{*}{ TEÓRICO } & Mínimo - Máximo & $4-10$ & $7-11$ & $<0,001$ \\
\hline & Media $\pm D e s v$. Típica & $7,4 \pm 1,7$ & $9,3 \pm 1,7$ & \\
\hline \multirow[t]{2}{*}{ PRAGMÁTICO } & Mínimo - Máximo & $4-10$ & $8-11$ & 0,109 \\
\hline & Media $\pm D e s v$. Típica & $7,6 \pm 2,2$ & $9,4 \pm 15$ & \\
\hline \multirow[t]{2}{*}{ Total } & Mínimo - Máximo & $4-10$ & $7-12$ & $<0,001$ \\
\hline & Media $\pm D e s v$. Típica & $7,5 \pm 1,5$ & $9,3 \pm 1,3$ & \\
\hline
\end{tabular}


Elaboración del mapa conceptual sin y con Cmap Tools utilizando la rúbrica semántica de Miller-Cañas 2008 Los puntajes deben variar entre 1 y 16. Los criterios a evaluar fueron:

\begin{tabular}{|l|l|l|}
\hline \multicolumn{2}{|c|}{ CRITERIOS DE EVALUACIÓN DE LA RÚBRICA SEMÁNTICA DE MILLER-CAÑAS 2008 } \\
\hline $\begin{array}{l}\text { Relevancia y ex- } \\
\text { haustividad de los } \\
\text { conceptos }\end{array}$ & $\begin{array}{l}\text { Nos indica la presencia de conceptos principales, el estilo teórico y reflexivo sobresalió mostran- } \\
\text { do un 50\% de los conceptos relevantes en el pretest y postest respectivamente. }\end{array}$ & Puntaje: \\
\hline $\begin{array}{l}\text { Estructura propo- } \\
\text { sicional }\end{array}$ & $\begin{array}{l}\text { Representa la unidad de significado que nos transmite alguna información según 2 conceptos } \\
\text { y un enlace con algunas excepciones en que se requiere } 3 \text { conceptos. El estilo activo destacó } \\
\text { en realizar proposiciones estructurales (-50\%) en el mapa conceptual sin Cmap Tools. El estilo } \\
\text { teórico en el mapa conceptual con Cmap Tools tuvo 1 o 2 proposiciones mal estructuradas. }\end{array}$ & $\begin{array}{l}\text { Los entre 1 y 16 } \\
\text { variar }\end{array}$ \\
\hline $\begin{array}{l}\text { Prop os icio nes s } \\
\text { erróneas }\end{array}$ & $\begin{array}{l}\text { Se refiere a contenidos de las proposiciones en los que se asigna un valor de verdad en referen- } \\
\text { cia a un objetivo, sin considerar aquellas proposiciones mal estructuradas que conduzcan propo- } \\
\text { siciones erróneas. En el pretest y postest destacó el estilo activo pasando de 1 o 2 proposiciones } \\
\text { erróneas a ninguna proposición errónea. }\end{array}$ & $\begin{array}{l}\text { Muy bajo (1-5) } \\
\text { Bajo (6-8) }\end{array}$ \\
\hline $\begin{array}{l}\text { Proposiciones } \\
\text { Dinámicas }\end{array}$ & $\begin{array}{l}\text { Expresan ideas con movimiento, acción, cambio de estado o relaciones de dependencia. Estas } \\
\text { pueden ser causativas y no causativas. Solo en el postest el estilo pragmático logró 1 a 2 pro- } \\
\text { posiciones causativas. }\end{array}$ & $\begin{array}{l}\text { Intermedio (9-11) } \\
\text { Alto (12-14) }\end{array}$ \\
\hline Enlaces cruzados & $\begin{array}{l}\text { Es la interrelación del concepto raíz con 2 conceptos, de tal manera que forman un circuito ce- } \\
\text { rrado. En el pre y postest no hubo enlaces cruzados. }\end{array}$ & Muy alto (15-16) \\
\hline $\begin{array}{l}\text { Jerarquía de } \\
\text { conceptos }\end{array}$ & $\begin{array}{l}\text { Es la estructuración jerárquica de los conceptos según su importancia. El estilo reflexivo logró } \\
\text { una jerarquía regular a buena en el postest. }\end{array}$ & \\
\hline
\end{tabular}

La puntuación mínima para todos los estudiantes pasó de 2 a 5 y la máxima de 9 a 12 puntos; ninguno alcanzó los 16 puntos. $\mathrm{Al}$ comparar la evaluación semántica antes y después del Cmap Tools, se muestra que antes de la ca- pacitación con Cmap Tools la puntuación fue de 4,5 $\pm 1,7$ para el total de la muestra y después pasó a $9,1 \pm 1,7$, siendo este incremento estadísticamente significativo $(p<0,05)$. Se observó este incremento significativo en los distintos estilos de aprendizaje, a excepción del estilo pragmático que también mejoró de un nivel semántico, muy bajo a un nivel semántico intermedio pero sin significancia estadística (Tabla 02).

Tabla 02. Comparación del nivel de la comprensión lectora antes y después de utilizar el Cmap Tools usando el criterio de la evaluación semántica Miller-Cańas (2008)

\begin{tabular}{|c|c|c|c|c|}
\hline \multirow{2}{*}{$\begin{array}{l}\text { ESTILOS DE } \\
\text { APRENDIZAJE }\end{array}$} & \multirow{2}{*}{$\begin{array}{l}\text { ESTADISTICAS } \\
\text { DESCRIPTIVAS }\end{array}$} & \multicolumn{2}{|c|}{$\begin{array}{c}\text { Evaluación Semántica del Mapa } \\
\text { Conceptual }\end{array}$} & \multirow{2}{*}{$\mathbf{p}\left({ }^{*}\right)$} \\
\hline & & $\begin{array}{c}\text { Antes } \\
\text { Cmap Tools }\end{array}$ & $\begin{array}{c}\text { Después } \\
\text { Cmap Tools }\end{array}$ & \\
\hline \multirow[t]{2}{*}{ ACTIVO } & Mínimo - Máximo & $3-8$ & $7-12$ & \multirow{2}{*}{$<0,001$} \\
\hline & Media $\pm D e s v$. Típica & $4,5 \pm 1,5$ & $9,3 \pm 1,5$ & \\
\hline \multirow[t]{2}{*}{ REFLEXIVO } & Mínimo - Máximo & $2-7$ & $6-12$ & \multirow{2}{*}{0,011} \\
\hline & Media $\pm D e s v$. Típica & $4,3 \pm 2,1$ & $8,9 \pm 1,9$ & \\
\hline \multirow[t]{2}{*}{ TEÓRICO } & Mínimo - Máximo & $2-9$ & $5-12$ & \multirow{2}{*}{$<0,001$} \\
\hline & Media $\pm D e s v$. Típica & $4,5 \pm 1,8$ & $9,2 \pm 1,6$ & \\
\hline \multirow[t]{2}{*}{ PRAGMÁTICO } & Mínimo - Máximo & $3-8$ & $6-12$ & \multirow{2}{*}{0,078} \\
\hline & Media $\pm D e s v$. Típica & $5,0 \pm 2,0$ & $8,6 \pm 2,3$ & \\
\hline \multirow[t]{2}{*}{ TOTAL } & Mínimo - Máximo & $2-9$ & $5-12$ & \multirow{2}{*}{$<0,001$} \\
\hline & Media $\pm D e s v$. Típica & $4,5 \pm 1,7$ & $9,1 \pm 1,7$ & \\
\hline
\end{tabular}

${ }^{*}$ ) Prueba estadística no paramétrica de Wilcoxon para muestras dependientes

\section{Discusión}

\section{La comprensión lectora sin Cmap Tools}

El test de comprensión lectora resultó regular. Los conceptos emitidos por los estudiantes son frases características de un aprendizaje memorístico, siendo propio de la falta de identificación de la idea principal, tal como mencionara García \& Díaz ${ }^{5}$, la estructura proposicional es inadecuada, hay mayormente proposiciones erróneas. No hubo enlaces cruzados, lo cual es indicativo de la falta de creatividad y de la capacidad de autopreguntarse; no hubo preguntas de enfoque, característico de los estudiantes que recién se inician en la elaboración de mapas conceptuales. También se observó una pobre organización jerárquica en aquellos que tienen un aprendizaje memorístico.

\section{La comprensión lectora con Cmap Tools:}

Los conceptos tuvieron mayor nivel semántico, se dio una mayor apropiación de conceptos relevantes, mayor diferenciación y reconciliación integradora, como también mayor capacidad de comprensión y mejor estructuración del mapa conceptual. Díaz ${ }^{3}$ mencionó, que extraer, relacionar y organizar jerárquicamente los conceptos principales es igual a una mejor estructuración de los mapas conceptuales y un mejor desempeño cognitivo.

Los alumnos crearon su propias proposiciones (unidad mínima de significa- do) demostrando apropiación de conceptos; tal como mencionara Manzano \& Aguilar ${ }^{6}$.

Las proposiciones erróneas: disminuyeron, se sugiere tener en cuenta la palabra de enlace y como lo mencionara Manzano \& Aguilar $^{8}$ la reelaboración del mapa y la autopregunta nos permite modificar el mapa conceptual a lo Miller ${ }^{7}$ menciona, es el resultado de la comprensión de lectura y dificultades del procesamiento de la información.

Proposiciones dinámicas causativas: Este punto no llegó a explicarse pero era de esperarse.

No hubo enlaces cruzados por falta de autopreguntarse cuál es la relación 
entre conceptos y a falta de facilitadores que incidieran en este punto.

Jerarquía: mejor estructuración del mapa conceptual con adecuada organización jerárquica pasando de proposiciones lineales a proposiciones claras y adecuadas. Si hay mayor nivel de habilidades lectoras, hay mejor orden jerárquico, mejor interrelación con los conocimientos en su proceso de comprensión dejando atrás la jerarquía lineal ${ }^{8}$.

\section{Conclusiones}

El uso de Cmap Tools favoreció la comprensión lectora de los estudiantes de pregrado de la Facultad de Odontología de la Universidad Nacional Mayor de San Marcos, observándose significancia estadística para todos los estilos de aprendizaje, menos para el estilo pragmático.

El estilo de aprendizaje predominante entre los estudiantes fue el estilo teórico.

\section{Referencias bibliográficas}

1. Briceño L. A., Rojas Velásquez, \& Peinado S. Influencia de los mapas conceptuales y los estilos de aprendizaje en la comprensión de la lectura. Revistas Estilos de Aprendizaje [Internet]. 2011 [Citado 2013-12-03]; 8(8):p.1-20. Disponible en: http://www.uned. es/revistaestilosdeaprendizaje/ numero_8/articulos/lsr_8_articulo_1.pdfdf

2. Roger S., Aguilar M. \& Manzano J. Efectos de un apoyo mediacional en tareas de elaboración de mapas conceptuales en estudiantes universitarios con diferentes niveles de habilidades lectoras. En Sánchez J. Cañas A. Novak J. D. editors.
Concept Maps: Making Learning Meaningful Proc. of Fourth Int. Conference on Concept Mapping Viña del Mar, Chile, 2010. [Citado 2013-12-03]. Disponible en: http://cmc.ihmc.us/cmc2010Proceedings/cmc2010\%20-\%20 Vol\%201.pdf

3. Díaz G. El papel de los mapas conceptuales en la organización del pensamiento de los estudiantes. En Sánchez J. Cañas A. Novak J. D. Editors Concept Maps: Making Learning Meaningful Proc. of Fourth Int. Conference on Concept Mapping J. Sánchez, A.J.Cañas, J. D. Novak, editors. Viña del Mar, Chile, 2010. [Citado 2013-12-03]. Disponible en: http://cmc.ihmc.us/cmc2010Proceedings/cmc2010\%20-\%20 Vol\%201.p

4. Correia P. \& Guilares J. Concept mapping informed by cognitive load theory: implications for tasks involving learner-generated cmaps. En Correia P. Malachias M. Cañas A. J. Novak J. D. editors. Concept Mapping to Learn and Innovate Proc. of Sixth Int. Conference on Concept Mapping Santos, Brazil, 2014. [Citado 201312-03]. Disponible en: http://cmc. ihmc.us/cmc2014Proceedings/ cmc2014\%20-\%20Vol\%201.pdf

5. García D \& Díaz A. Uso de mapas conceptuales en estudiantes universitarios de nuevo ingreso. En Correa P. Malachias M. Cañas A. J. Novak J. D. editors Concept Mapping to Learn and Innovate Proc. of Sixth Int. Conference on Concept Mapping Santos, Brazil, 2014. [Citado 2013-12-
03] Disponible en: http://cmc. ihmc.us/cmc2014Proceedings/ cmc2014\%20-\%20Vol\%202.pdf

6. Manzano C. J. \& Aguilar T. M. F. El uso del mapa conceptual para la comprensión de textos. En Paulo R. M. Correia María E. I. Malachias Cañas A. J.Novak J.D. editors. Concept Mapping to Learn and Innóvate Proc. of Sixth Int. Conference on Concept Mapping Santos, Brazil, 2014 Volume 1, p.233-239. [Citado 2013-12-03]. Disponible en: http://cmc.ihmc. us/cmc2014/cmc2014Program. html

7. Miller L. \& Cañas, A. J. “An exploration of computer-mediated skill acquisition in concept mapping by in-service Panamanian public elementary schoolteachers" [Disertación] tesis doctoral Universitat Oberta de Catalunya 2008. [Citado 2013-12-03]. Disponible en:http://184.182.233.151/ rid\%3D1J6Z2SV24-1TZ7JP01DNL/Norma\%2520Miller\%252 0\%2520Doctoral\%2520. Dissertation\%252025-03-08.pdf

8. Roger S., Aguilar M. \& Manzano J. Efectos de un apoyo mediacional en tareas de elaboración de mapas conceptuales en estudiantes universitarios con diferentes niveles de habilidades lectoras. En Sánchez J. Cañas A. Novak J. D. editors. Concept Maps: Making Learning Meaningful Proc. of Fourth Int. Conference on Concept Mapping Viña del Mar, Chile, 2010. [citado 2013-12-03]. Disponible en: http://cmc.ihmc.us/cmc2010Proceedings/cmc2010\%20-\%20 Vol\%201.pdf 